\title{
Testing vaccines in the time of Covid: The changing landscape
}

\author{
RICHARD A CASH
}

\begin{abstract}
The initial trials of SARS-CoV-2 vaccines were randomised control trials (RCT) with a placebo as control. The use of a placebo was ethically justified because, as with any new and emerging infectious disease, there was no known vaccine. There are now at least eight vaccines that have been shown to be effective and approved for emergency use, so the use of a placebo in the control group is no longer ethically justified. This article discusses why ethical guidelines should be continually evaluated in a changing landscape and why trust is so important.
\end{abstract}

Key words: Covid vaccine, RCTs, placebo controls, research ethics,

A recent review by a World Health Organisation (WHO) Ad Hoc Expert Group (1), made the case for placebo-controlled trials of Covid-19 vaccines to continue. They argued that there is a need to obtain the following information: assessment of shorter and longer-term safety data; duration of immunity; the impact of waning immunity on protection, and possible vaccine enhanced disease from SARS-CoV 2; protection against clinically severe disease; and the association between degree of protection and age of recipient as well as co-existing conditions. The emergence of new variations of the virus (the UK, South Africa, Brazil, etc) call for additional vaccine studies. This information should be collected on both older and newer vaccines. A similar review, suggesting alternatives/strategies for testing vaccines has recently been published by Eyal and Lipsitch (2)

Whenever a new disease emerges, there are ethical dilemmas regarding the testing of vaccines, new drugs, and new methods of care. The disease has yet to be fully described, treatments may be unknown, and the epidemiology is being defined in real time. Baseline data is limited. The gold standard of study design remains the randomised placebo-control

Author: Richard A Cash (racash@hsph.harvard.edu), Senior Lecturer, Department of Global Health and Population, Harvard T.H. Chan School of Public Health, Boston, Massachusetts, USA.

To cite: Cash RA. Testing vaccines in the time of Covid: The changing landscape. Indian J Med Ethics. 2021 Apr-Jun; 6(2) NS: 128-129. DOI: 10.20529/ IJME.2021.018.

Published online first on March 15, 2021.

Manuscript Editors: Ruth Macklin, Amar Jesani.

Peer reviewer: An anonymous reviewer.

(c) Indian Journal of Medical Ethics 2021 study. In early stages of discovery there is no standard therapy or vaccine(s), so the use of a placebo is justifiable. This has been the case with Covid-19.

Trials dealing with therapeutics have focused on testing established drugs for other purposes rather than on the development of new products. For vaccines, the story has been different. The new biology has vastly accelerated the development and testing of vaccines within a time frame that has been nothing short of remarkable. From the time that the genome was released by Chinese scientists in January 2020 until the first vaccine was developed, it took only 10 weeks. Research methods developed over many years created platforms for vaccine development as soon as the novel virus was characterised.

The first vaccines were produced by Moderna and BioNTech (which partnered with Pfizer), both using the mRNA technology. Phase 3 trials for each, comparing the vaccine to placebo (with a combined total of 75,000 subjects) were completed in October and November 2020 and the vaccines were approved for Emergency Use by the US Food and Drug Administration (FDA) in December. A number of other vaccine candidates using different technologies were also being tested in Phase 1, 2, 3 studies and applications have been submitted for approval to the US FDA as well as to other national governmental agencies. These include the following: Johnson and Johnson; Novavax; Oxford-AstraZeneca (Covishield); Sputnik V (Russia); Sinopharm and Sinovac (China); and Covaxin (India). There are almost 200 other vaccine candidates in different stages of development.

The continued development and testing of vaccines has led to ethical dilemmas which include the following $(1,2)$ : (i) Should the control group receive a vaccine or placebo in future vaccine studies? (ii) What information should vaccinees or control groups receive prior to the study regarding study design and access to a vaccine shown to be effective? (iii) Is it appropriate to continue blinded follow up of placebo recipients in existing trials and should trial sponsors be obligated to unblind treatment assignments for participants who desire a vaccine?

If an effective vaccine is licensed should all future vaccine candidates be compared to this vaccine? How is "effective" defined and who makes this determination: The WHO? A 
national scientific body? What if the comparative vaccine is not available in the country where a trial is being undertaken? This could occur because of cost or the ability to maintain conditions to ensure vaccine viability, such as storing a vaccine in subzero temperatures required by the Pfizer/BioNTech vaccine? A vaccine requiring only one dose and/or which can be stored at room temperature, would be far more attractive to low-and middle-income countries (LMIC). Should this vaccine be compared only with a similar product meeting these criteria?

Since the statement from the WHO Ad Hoc Expert Group was developed in early November and first published in early December 2020 (1), much has changed. A number of effective vaccines (70-95\% against clinical disease) have been licensed under Emergency Use or Compassionate Use provisions. One or more of these vaccines are now potentially available anywhere in the world, even if supplies may be limited. Public health recommendations have been published by the WHO as well as many national public health authorities. As noted, early on in the pandemic there were no effective vaccines so the use of a placebo comparator was justified. But new and emerging diseases are like a flowing river. Information is constantly changing; the ethical parameters must change as well. At this moment, it seems difficult to justify the use of a placebo during a vaccine trial using any of the WHO criteria for exceptions (3). Though mortality is highest in those over 60 (and in certain other high-risk groups), morbidity and mortality still occur in younger age groups, so the age of the participants should not be factored into this decision. The risk is not negligible so, from this time forward, vaccine studies will have to be designed to compare a new vaccine with one that is already approved (even if only under emergency use authorisation). This will determine whether the newly developed vaccine is as safe and effective as those already in use. By not including a placebo group, the duration and expense of trials will certainly increase (4). Some might argue that in testing a vaccine against a new variant of SARS-CoV-2 the use of a placebo in the control group would be justified; but some of the current vaccines appear to be effective in preventing severe disease from new strains that may be more infectious.

As in all research with human beings, attention to the informed consent process and document is critically important. Study participants should be given all relevant information as to the effectiveness of the product being tested, possible side effects, compensation if adverse conditions occur, and the ability to withdraw from the study at any time without prejudice. Should a vaccine prove to be effective, volunteers should be told and be allowed to choose as to whether to have the test vaccine or another proven product. Even if the participant is not in a group prioritised to receive the vaccine, half of the study group will have received the vaccine and so should the controls. The informed consent document should be clear on these issues and understood by the participant (a quiz to determine understanding could be given). All effort should be made to follow up cases and controls for the very reasons stated by the committee.

What information should be available to trial participants after the study has been completed? Is it fair or ethical to not inform a trial participant what vaccine they have received in the trial? If it is possible that vaccines based on different approaches might interfere with each other, shouldn't a person be made aware of this? As of now, there are no studies on whether vaccines interact with each other (5). If the participant wishes to use a vaccine other than the first vaccine (or placebo), they should have the information on which to make an informed choice. As an example, Moderna unblinded its trial and informed participants if they were in the placebo arm, giving them the option to obtain the vaccine. Long-term comparison of vaccine to placebo was no longer possible, but there was the opportunity to increase safety data and obtain long-term information on efficiency.

Public health authorities are rightly concerned that many will refuse the vaccine, a response often referred to as vaccine hesitancy. The hesitancy will put many individuals at risk of infection and could limit overall immunity in the population. Reasons given for vaccine hesitancy include religious beliefs, politics, false news reports (part of the infodemic landscape) and misunderstanding of both individual and community risk. An underlying reason given for hesitancy is a lack of trust in government, science, and public health authorities. It is critical, therefore, that pharmaceutical companies, drug regulators, government, and the public health community develop and nurture trust. If the public feels information is withheld and study participants are not being fully informed, trust will be damaged and no amount of data will be able to overcome this mistrust, especially in the short run. Plummeting rates of measles vaccination and increased measles cases followed the dengue vaccine controversy in the Philippines (6). The research community should ensure that quality ethical science be conducted in the testing of new vaccines and information is clear and transparent, if these vaccines are to find their way into the arms of the public.

\section{References}

1. WHO Ad Hoc Expert Group on the Next Step for Covid-19 Vaccine Evaluation. Placebo-Controlled Trials of Covid-19 Vaccines-Why we Still Need Them. N Engl J Med. 2021 Jan 14; 384(2):e2(1)-e2(3).Epub 2020 Dec 2.

2. Eyal N. Lipsitch $M$, How to test SARS-CoV-2 vaccines ethically even after one is available. Clin Infect Dis. 2021 Feb 26; ciab182. doi: 10.1093/ cid/ciab182. Online ahead of print.

3. Rid A, Saxena A, Baqui AH, Bhan A, Bines J, Bouesseau M-C, et al. Placebo use in vaccine trials: recommendations of a WHO expert panel. Vaccine. 2014 Aug 20; 32(37): 4708-12: Epub 2014 Apr 25.

4. Cohen J. First vaccine may stymie hunt for better ones, Science. 2020 Oct 3[cited 2021 March 6]; 370(6515): 389-90. Available from; https:// science.sciencemag.org/content/370/6515/389

5. World Health Organisation. COVID-19 Vaccines: Surveillance Safety Manual. Geneva, Switzerland; World Health Organization: 2021[cited 2021 Mar 6]. Available from:https://www.who.int/vaccine_safety/ committee/Module_Establishing_surveillance_systems.pdf

6. Beaubien J. The Philippines is fighting one of the world's worst measles outbreaks. NPR News. 2019 May 23[cited 2021 Mar 6]. Available from: https://www.npr.org/sections/goatsandsoda/ 2019/05/23/725726094/the-philippines-is-fighting-one-of-the-worldsworst-measles-outbreaks 